\title{
Elevated Urinary Methylmalonic Acid/creatinine ratio and Serum Sterol levels in Patients with Acute Ischemic Stroke
}

\author{
Abdullah Sivrikaya1, Serefnur Ozturk², Hakan Ekmekci², Aslıhan Sağlam², \\ Sedat Abusoglu ${ }^{1 *}$, Ali Unlu ${ }^{1}$ \\ 1. Department of Biochemistry, Selcuk University Faculty of Medicine, Turkey \\ 2. Department of Neurology, Selcuk University Faculty of Medicine, Turkey
}

\begin{abstract}
Introduction: Sitosterolemia, defined as phytosterolemia, is a rare autosomal recessive disease characterized by elevated blood sterol levels. Our aim was to investigate serum plant sterols, methylmalonic acid, vitamin B12, oxidized-LDL and homocysteine levels in ischemic and hemorrhagic stroke patients and healthy subjects.

Material and Methods: 50 healthy subjects (without a family history of coronary artery disease) and 89 patients hospitalized in the Selcuk University neurology clinic or intensive care unit with a diagnosis of stroke were included in this study. Serum plant sterols, homocysteine and methylmalonic acid, oxidized-LDL, total cholesterol, triglycerides, HDL-Cholesterol and vitamin B12 levels were analyzed by gas chromatography-mass spectrometry, liquid-chromatography tandem mass spectrometry, commercially available ELISA kit, spectrophotometry and chemiluminescence methods, respectively.

Results: Urinary methylmalonic acid/creatinine ratio $(p<0.05)$, serum $\beta$-sitosterol levels and $\beta$-sitosterol/ cholesterol ratio were significantly higher $(p<0.01)$ in patients compared to the control group. There was a significant positive correlation between the serum OxLDL-methylmalonic acid, serum homocysteine- urinary methylmalonic acid /creatinine ratio, serum methylmalonic acid - Urinary methylmalonic acid $(p<0.05)$, serum homocysteine- urinary methylmalonic acid, urinary methylmalonic acid-methylmalonic acid/creatinine ratio, serum methylmalonic acid-methylmalonic acid/creatinine ratio, serum beta-sitosterol-beta-sitosterol/cholesterol, total cholesterol-HDL, total cholesterol-LDL $(p<0.01)$ levels and negative correlation between vitamin B12- serum methylmalonic acid ( $p<0.05)$, cholesterol-stigmasterol/cholesterol, LDL-stigmasterol/cholesterol $(p<0.01)$ levels in the patient group.
\end{abstract}

Conclusion: Our findings presented that the serum sitosterol levels were significantly higher in stroke patients compared to controls.

Keywords: sterol, stroke, oxidized LDL, methylmalonic acid, creatinine

Received: 24 $4^{\text {th }}$ August 2017; Accepted: $5^{\text {th }}$ December 2017; Published: $17^{\text {th }}$ December 2017

\footnotetext{
*Corresponding author: Sedat Abusoglu, Department of Biochemistry, Selcuk University Faculty of Medicine, Turkey. E-mail: sedatabusoglu@yahoo.com
} 


\section{Introduction}

Stroke is the third cause of mortality in the world. According to the World Health Organization (WHO), about 5.71 million people suffered from stroke in 2004, and it is estimated that this number will increase to 6.3 and 7.8 million in 2015 and 2030, respectively [1].

Statins are recommended as a cholesterol-lowering agent in developing countries. Plant sterols are used as alternative treatment protocol. Firstly, sterols were inserted in margarine and used in Europe in 1997. The most frequent phytosterols in the human diet are $\beta$-sitosterol, campesterol and stigmasterol, (almost 65\%, 30\% and $3 \%$ of diet ingredients, respectively). The phytosterols to limit the absorption of cholesterol were first recognized in 1953 [2].

Although the chemical structure of phytosterols are very similar to cholesterol, they are differentiated according to differences in the cholesterol side-chain group [3-6]. Their cholesterol-lowering properties are not well-known. They compete with cholesterol for dissolution in the micelles and therefore inhibit intestinal absorption of both dietary and endogenous cholesterol [6].

Phytosterols cannot be synthesized by humans and, therefore, should be avoided from the diet. Over 40 phytosterols have been defined; of these, stigmasterol, campesterol, and $\beta$-sitosterol constitute more than $95 \%$ of total dietary intake [7].

Sitosterolemia, defined as phytosterolemia, is a rare autosomal recessive disease characterized by elevated blood sterol levels, induced by raised intestinal absorption and reduced biliary drain of plant sterols. Sitosterolemia is due to mutations of some ATP-binding cassette (ABC), ABCG5 and ABCG8 protein genes. ABC transport proteins reduce the sterol absorption in intestinal cells by pumping back the plant sterols into the intestinal lumen [8].
Vitamin B12 and folate deficiency, especially in population over the age of 55, leads to increased levels of serum methylmalonic acid and homocysteine concentrations. Increased methylmalonic acid levels in the urine were found to be associated with ischemic stroke [9].

Homocysteine is considered to play a role in the pathogenesis of ischemic stroke. Homocysteine (Hcy) is a cause of atherogenesis and thrombogenesis due to endothelial damage, coagulation disorders and vascular smooth muscle proliferation [10].

Low-density lipoprotein cholesterol (LDL-cholesterol) contains not only cholesterol, but also plant sterols (i.e., campesterol, sitosterol, and avenasterol) [11]. The former stages of oxidized LDL (Ox-LDL) formation is the peroxidation of polyunsaturated fatty acids in its structure [12].

Plasma stanol levels were found to decrease sterol levels but increase the hemorragic stroke risk compared to sterols in hypertensive rats vulnerable to stroke [13]. Urinary methylmalonic acid levels were found to be associated with ischemic stroke [9]. Low serum High density lipoprotein cholesterol (HDL-cholesterol) and triglyceride levels have been concluded to be related with post-acute ischemic stroke mortality [14].

The aim of this study was to investigate serum plant sterols, methylmalonic acid, vitamin B12, Ox-LDL and homocysteine levels in ischemic and hemorrhagic stroke patients and healthy subjects. To the best of our knowledge, this study is the first on Turkish population.

\section{Material and Methods}

\section{Study Population}

This study was performed on 50 healthy subjects (26 male, 24 female) aged between 5664 years (without a family history of coronary artery disease) and 89 patients (49 male, 40 female) aged between 54-69 years hospitalized in 
the Selcuk University neurology clinic or intensive care unit with a diagnosis of stroke. Patients with steroid therapy for acute stroke, with a medication history of anti-hyperlipidemic drug, alcohol consumption, vegetarian diet, malignancy, infection, end-stage liver and kidney disease, vitamin B12 and folic acid therapy were excluded. We obtained $8-10 \mathrm{ml}$ of whole blood and urine samples from patients after 12-14 hours of fasting on the first day. Serum samples were immediately seperated after coagulation and stored at $-85^{\circ} \mathrm{C}$ until the day of analysis. Serum total cholesterol, triglycerides, HDL-cholesterol, LDL-cholesterol, vitamin B12, plant sterols (stigmasterol, $\beta$-sitosterol), Ox-LDL, serum methylmalonic (SMMA), urinary methylmalonic acid (UMMA) and homocysteine levels were analyzed. The study was approved by Selcuk University non-invasive Clinical Research Ethics Committee (Number of approval: 2013/07)

\section{Laboratory Procedures}

Serum Ox-LDL levels were determined by using a commercially available kit (Mercodia Oxidized-LDL ELISA kit, Cat no: 101143-01). Serum total-cholesterol, HDL-cholesterol and triglyceride levels were analyzed using a commercially available kit (Abbott cat no:91752UN16, 48507UQ10, 12603UN16, respectively) on Abbott Architect c16000 autoanalyzer. LDL-cholesterol levels were calculated by Friedewald Formula. Serum vitamin B12 levels were measured using a commercially available kit (Roche; cat no:11427004) on Roche Cobas e-170 autoanalyzer with chemiluminescence method. Serum homocysteine levels were determined by LC-MS/MS (Liquid Chromatography Tandem Mass Spectrometry) as previously described by Rafii et al. [16] with minor modifications. Briefly, $50 \mu \mathrm{L}$ plasma, calibrator and control samples were mixed with $50 \mu \mathrm{L}$ internal standard $(10 \mu \mathrm{M}$ d8-homocysteine isotope DLM-3619-1) and $50 \mu \mathrm{L}$ reducing reagent
(300 mmol/L 1,4-Dithiothreitol) and incubated at room temperature for 15 minutes. $300 \mu \mathrm{L}$ of precipitating reagent $(15 \%$ trichloroacetic acid Cat No: Merck 100810) was added to precipitate proteins, mixed for 10 seconds and centrifuged at $13.000 \mathrm{rpm}$ for 3 minutes. $10 \mu \mathrm{L}$ of supernatant was injected into the analytical column for chromatography (Figure 1).

Serum and urinary methylmalonic acid levels were determined by LC-MS/MS (Liquid Chromatography Tandem Mass Spectrometry) as previously described by Kushnir et al. [17] with minor modifications. Briefly, $100 \mu \mathrm{L}$ of internal standard $(2 \mu \mathrm{M} d 3$ - methylmalonic acid) in water was incorporated in $1000 \mu \mathrm{L}$ standard, serum and urine $(0.1 \mathrm{~mL}$ of specimen and 0.9 $\mathrm{mL}$ of water) and $3 \mathrm{ml}$ methyl tert butyl ether (MTBE) (containing $30 \mathrm{ml} / \mathrm{L}$ of phosphoric acid) and mixed for $5 \mathrm{~min}$ and centrifuged at $3000 \mathrm{rpm}$ for 10 minutes to avoid the precipitated proteins. The supernatant was collected and dried under a nitrogen gas flow, and $40 \mu \mathrm{L}$ of n-butanol including $3 \mathrm{~mol} / \mathrm{L} \mathrm{HCl}$ was added. The mixture was incubated at $60{ }^{\circ} \mathrm{C}$ for 35 minutes and dried under a nitrogen gas flow. The samples were diluted with $75 \mu \mathrm{L}$ of a mixture of methanol-0.005 mol/L ammonium formate $(1 / 1, \mathrm{v} / \mathrm{v})$ and transported to labeled autosampler vials and injected into analytical column for chromatography. Mass spectrometric assay was performed by Shimadzu LC-20-AD (Kyoto, Japan) coupled with a ABSCIEX API 3200 triple quadrupole mass spectrometer (USA) with an electrospray ion source (ESI) operating in positive mode. Results were calculated with Analyst software and processed for calibration and quantification of the analytes with Quantitation Wizard Analyst Software 1.6.1 by ABSCIEX Technical Services (USA) (Figure 2).

Serum plant sterol levels were determined by GC-MS (gas chromatography-mass spectrometry) as described by Ahmida et al. [15] with minor modifications. Briefly, $20 \mu \mathrm{L}$ of internal standard 

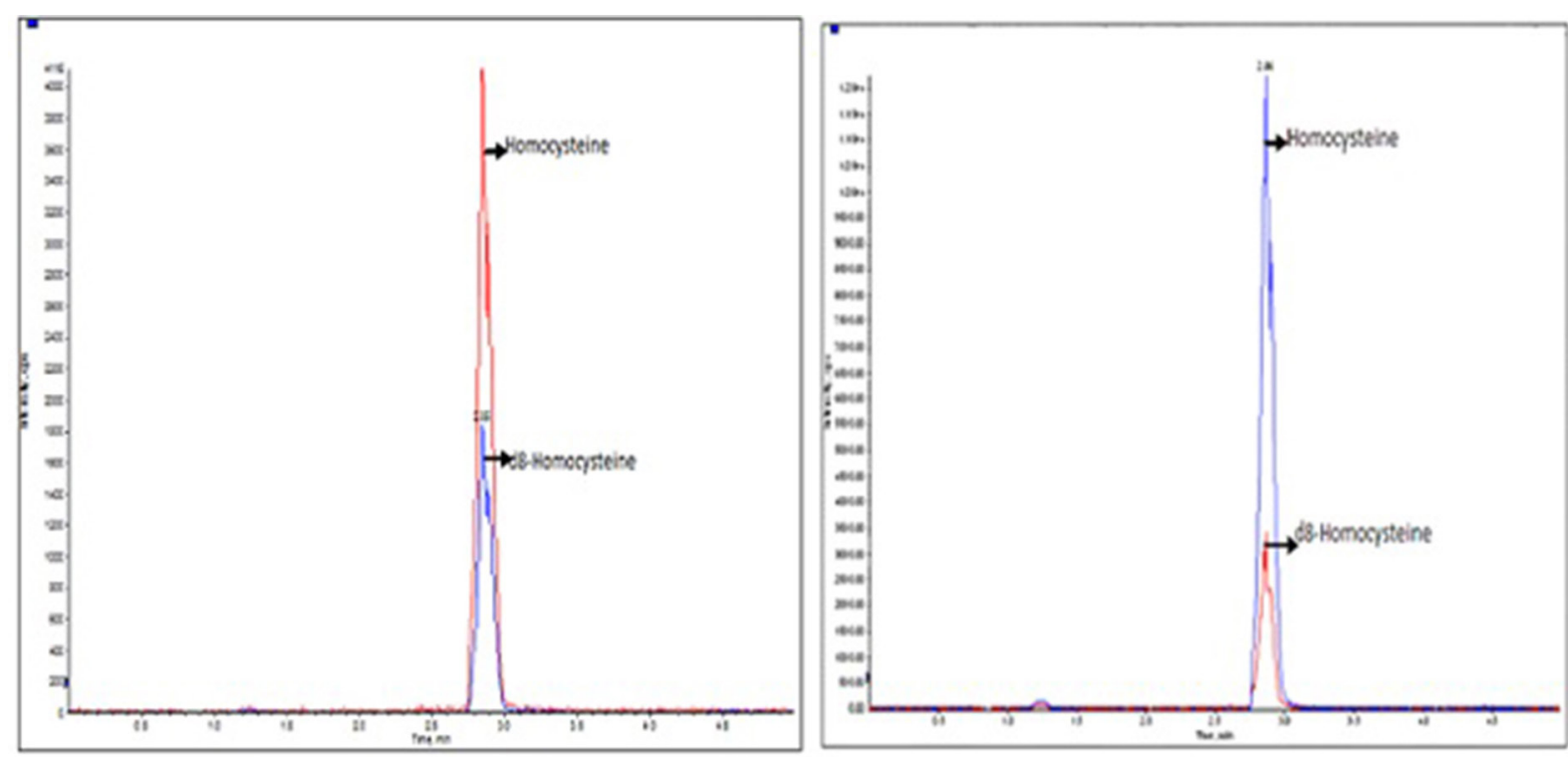

Fig. 1. The serum chromatograms of healthy (left) and patient (right) homocysteine for 10 and 50 $\mu \mathrm{mol} / \mathrm{L}$ of homocysteine, respectively.

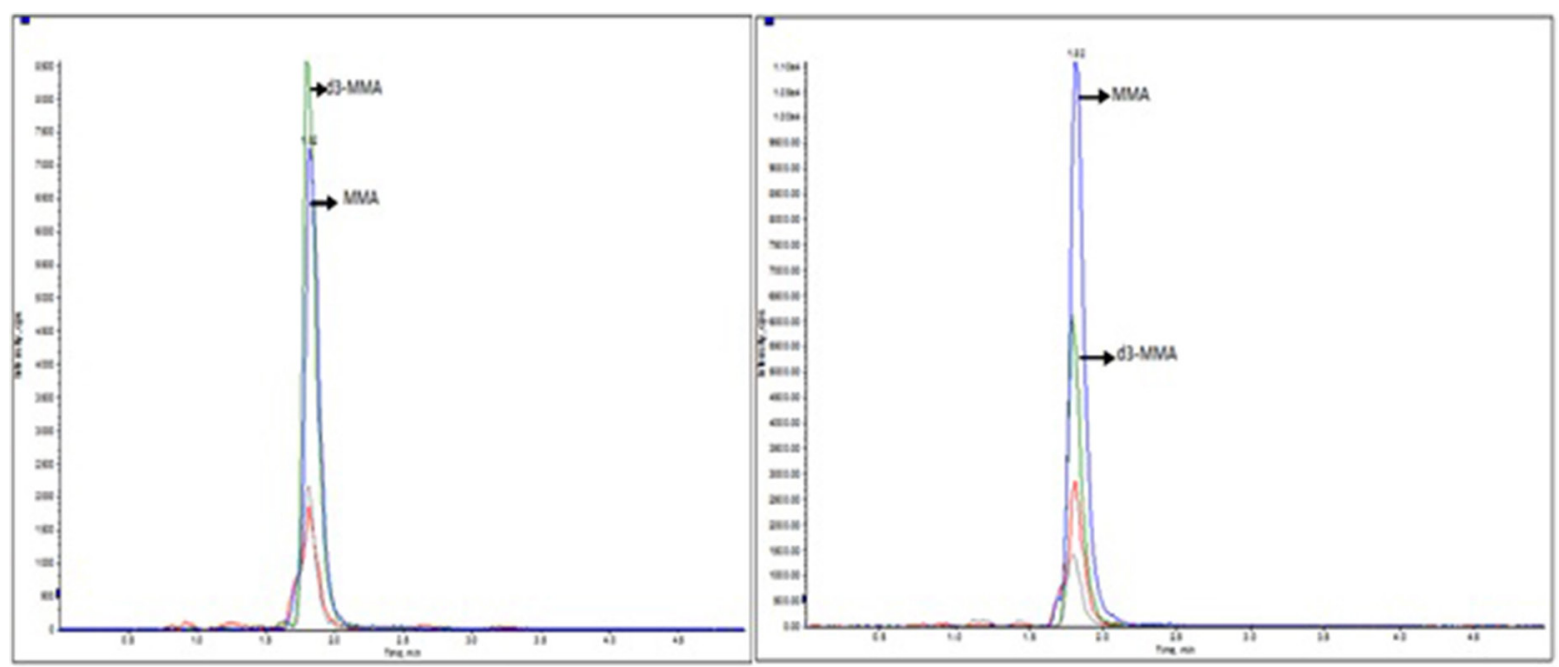

Fig. 2 . The urinary chromatograms of healthy (left) and patient (right) methylmalonic acid for 2.29 and $5.98 \mu \mathrm{mol} / \mathrm{L}$ methylmalonic acid, respectively.

(1000 $\mu \mathrm{M} 5 \alpha$-cholestane) in toluene were added to $200 \mu \mathrm{L}$ standard or plasma in glass tubes including $1 \mathrm{ml}$ of potassium hydroxide in ethanol $(1.0 \mathrm{~mol} / \mathrm{L})$. Samples were vortexed, dried with $\mathrm{N} 2$ and incubated at $70{ }^{\circ} \mathrm{C}$ for 60 minutes in the dark. The reactions were stopped by cooling the tubes under cold water. After refrigerating, water $(1 \mathrm{ml})$ was added and the lipids and extracted twice with $2 \mathrm{ml}$ of a solution of hexane and absolute ethanol (20/1, v/v), including 12.5 $\mathrm{mg} / \mathrm{L}$ butylated hydroxytoluene. The tubes were mixed and then centrifuged at $3500 \mathrm{rpm}$ at 20 
${ }^{\circ} \mathrm{C}$ for 10 minutes to provide phase separation; the organic phase was removed to small glass vials, flushed completely under a steam of N2. The lipid extract was derivatized with $200 \mu \mathrm{L}$ freshly prepared pyridine-N,O-Bis(trimethylsilyl)trifluoroacetamide (BSTFA) with $(1 / 1, \mathrm{v} / \mathrm{v})$. Specimens were then incubated at $70{ }^{\circ} \mathrm{C}$ for 60 minutes and analyzed with GC-MS (Figure 3).

\section{Statistical Analysis}

Statistical analysis was performed with SPSS v16. Student's t-test and Mann-Whitney U-test were used for parametric (Ox-LDL, homocysteine, vitamin B12, triglycerides, methylmalonic acid, stigmasterol, $\beta$-sitosterol and $\beta$-sitosterol/ cholesterol ratio) and non-parametric variables (cholesterol, HDL-cholesterol, LDL-cholesterol and stigmasterol/cholesterol ratio), respectively. Pearson and Spearman correlation tests were performed for parametric and non-parametric tests. Data were expressed as mean \pm (standard deviation) $(\bar{X} \pm \mathrm{SD})$. Results were considered statistically significant at $\mathrm{P}<0.05$.

\section{Results}

Laboratory results are presented in Table 1 and Table 2. UMMA levels of the patient group were significantly $(\mathrm{P}<0.01)$ lower than in the control group, UMMA/creatinine ratio $(\mathrm{P}<0.05)$, $\beta$-sitosterol and $\beta$-sitosterol/cholesterol ratio levels were significantly higher $(\mathrm{P}<0.01)$ in the patient group compared to controls. There was no statistically significant difference for other laboratory parameters.

There were significant positive correlations between the OxLDL-SMMA, homocysteine-MMA/creatinine ratio, SMMA-UMMA $(\mathrm{P}<0.05)$, homocysteine-UMMA, UMMA-MMA/creatinine ratio, SMMA-MMA/ creatinine ratio, Betasitosterol-Betasitosterol/ cholesterol ratio, cholesterol-HDL, cholesterol-LDL $(\mathrm{P}<0.01)$ levels and negative correlations between Vitamin B12-SMMA $(\mathrm{P}<0.05)$, cholesterol-stigmasterol/cholesterol ratio, LDL-stigmasterol/cholesterol ratio $(\mathrm{P}<0.01)$ levels in the patient group.

Significant positive correlations were also found between SMMA-UMMA $(\mathrm{P}<0.05)$, UMMA-MMA/creatinine ratio, Betasitosterol-Betasitosterol/cholesterol ratio, cholesterol-LDL $(\mathrm{P}<0.01)$ levels and negative correlations between the levels of cholesterol-HDL $(\mathrm{P}<0.05)$, cholesterol-stigmasterol/cholesterol ratio, HDLLDL and LDL-stigmasterol/cholesterol ratio $(\mathrm{P}<0.01)$ levels in the control group.

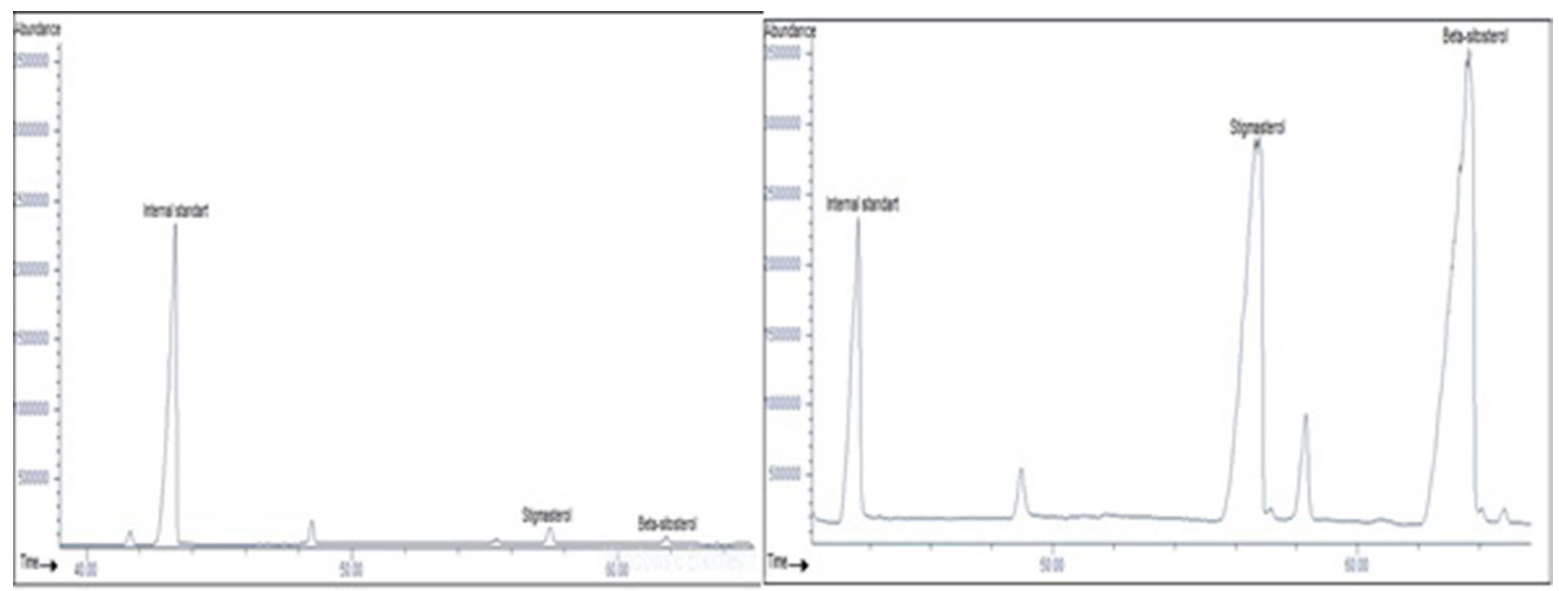

Fig. 3. The serum chromatograms of healthy (left) and patient (right) stigmasterol and Betasitosterol for $1.65 \mu \mathrm{g} / \mathrm{ml}, 2.78 \mu \mathrm{g} / \mathrm{ml}$ and $16.21 \mu \mathrm{mol} / \mathrm{L}, 25.24 \mu \mathrm{g} / \mathrm{ml}$, respectively. 
Table 1. Lipid parameters of both groups

\begin{tabular}{lccc}
\hline Parameters & Patients $(\mathrm{n}=89)$ & Controls $(\mathrm{n}=50)$ & $\mathrm{P}$ \\
\hline Total cholesterol $(\mathrm{mg} / \mathrm{dl})$ & $178(121-276)$ & $189(121-257)$ & 0.323 \\
\hline Triglyceride $(\mathrm{mg} / \mathrm{dl})$ & $147.78 \pm 89.57$ & $160.55 \pm 70.52$ & 0.433 \\
\hline HDL-cholesterol $(\mathrm{mg} / \mathrm{dl})$ & $35(17-64)$ & $40.0(23-74)$ & 0.010 \\
\hline LDL- cholesterol $(\mathrm{mg} / \mathrm{dl})$ & $114.4(30.6-199.2)$ & $109.6(24-182.4)$ & 0.839 \\
\hline
\end{tabular}

The values of triglyceride are expressed as mean \pm standard deviation.

The values of total cholesterol, HDL-cholesterol and LDL- cholesterol are expressed as median(min-max)

Table 2. The OxLDL, VitB12, UMMA, SMMA, Homocysteine, Stigmasterol, Beta-sitosterol levels; UMMA/Creatinine, Stigmasterol/cholesterol and Beta-sitosterol/cholesterol ratio of both groups.

\begin{tabular}{lccc}
\hline Parameters & Patient group $(\mathrm{n}=89)$ & Control grup $(\mathrm{n}=50)$ & $\mathrm{P}$ \\
\hline Age & $61.95 \pm 7.18$ & $60.08 \pm 4.65$ & 0.123 \\
\hline OxLDL $(\mu \mathrm{g} / \mathrm{L})$ & $1.87 \pm 1.42$ & $1.90 \pm 1.59$ & 0.193 \\
\hline VitB12 $(\mathrm{pg} / \mathrm{ml})$ & $353.90 \pm 223.99$ & $350.73 \pm 197.45$ & 0.936 \\
\hline UMMA $(\mu \mathrm{M})$ & $2.50 \pm 2.34$ & $4.51 \pm 3.21$ & 0.001 \\
\hline SMMA $(\mu \mathrm{M})$ & $0.57 \pm 0.33$ & $0.77 \pm 0.37$ & 0.063 \\
\hline Homocysteine $(\mu \mathrm{mol} / \mathrm{L})$ & $15.47 \pm 9.23$ & $13.95 \pm 5.53$ & 0.275 \\
\hline UMMA/Creatinine & $0.47 \pm 0.11$ & $0.45 \pm 0.04$ & 0.020 \\
\hline Stigmasterol $(\mu \mathrm{g} / \mathrm{ml})$ & $2.16 \pm 0.67$ & $2.25 \pm 0.53$ & 0.441 \\
\hline Beta-sitosterol $(\mu \mathrm{g} / \mathrm{ml})$ & $5.21 \pm 4.13$ & $3.12 \pm 0.67$ & 0.000 \\
\hline Stigmasterol $/ \mathrm{cholesterol}(\mu \mathrm{g} / \mathrm{mg})$ & $1.16(0.45-3.15)$ & $1.11(0.73-2.16)$ & 0.729 \\
\hline Beta-sitosterol $/ \mathrm{cholesterol}(\mu \mathrm{g} / \mathrm{mg})$ & $2.89 \pm 2.24$ & $1.77 \pm 0.62$ & 0.000 \\
\hline
\end{tabular}

OxLDL:Oxidized LDL; VitB12:Vitamin B12; UMMA:Urine methylmalonic acid; SMMA: Serum methylmalonic acid. The values of Stigmas terol/cholesterol and other parameters are expressed as median(min-max) and mean $\pm \operatorname{standard~deviation}(\bar{X} \pm \mathrm{SD})$, respectively.

\section{Discussion}

To the best of our knowledge, this is the first study investigating serum plant sterols, OxLDL, homocysteine, methylmalonic acid, vitamin B12 levels and correlations between these parameters in stroke patients.

Sitosterolemia is a genetic disease. Genetic factors, environmental factors and dietary habits may play an important role in the absorption of plant sterols. Therefore, the measurement of these sterols in our region's population is of great importance. Although there is no clinically important elevation of cholesterol levels, premature coronary heart disease has been reported to develop in patients with sitosterolemia [8]. In our study, $\beta$ - sitosterol and $\beta$ - sitosterol/cholesterol ratio levels of the patients were significantly higher compared to the control group. This phenomenon may increase the susceptibility to stroke. In addition, a significant positive correlation was found between betasitosterol- betasitosterol /cholesterol ratio levels in the patient group. This might be due to plant sterols to reduce the absorption of cholesterol at high levels. There is no other study investigating the levels of sitosterol in stroke patients.

Consumption of phytosterols has been reported to protect against heart attack and stroke. 
Phytosterols have anti-inflammatory, antibacterial, antifungal, antiulcerogenic and anti-tumoral activity [18]. Mammals do not synthesize phytosterols. Plant sterol supplementation disturbs endothelial function, exacerbates ischemic brain injury, effects atherogenesis in mice [19].

Ogawa et al. [20] have reported that the supplementation of phytosterol to soybean oil raised systolic blood pressure and supported the onset of stroke, which may cause reduced survival time in stroke-prone spontaneously hypertensive rats. Significant reduction in the intestinal mRNA expression of ABCG5 and ABCG8 might be responsible in stroke-prone spontaneously hypertensive rats.

Total cholesterol and LDL-cholesterol levels in the hypercholesterolemic patient group with a

6-week phytosterol consumption with lowfat fermented, natural plant sterol esters enriched milk were found to have significant reduction compared to control group [21]. Positive correlation was found between betasitosterol- betasitosterol /cholesterol ratio levels in the patients group. These findings were compatible with the resuts of our study.

It was reported that the homocysteine causes vascular damage [22] and induces procoagulant activity [23]. On the other hand, Kara et al. [9] have found no significant difference in serum vitamin B-12, folate and homocysteine levels between the patients with ischemic stroke and control groups. Urinary MMA concentrations and erythrocyte folate levels were significantly higher in patients compared to controls. In addition, urinary MMA/creatinine ratio was significantly higher in the patient group. These findings are not consistent with our results. In our study, there was no significant difference between patients and control groups for urinary MMA/creatinine ratio. The possible cause for this finding might be due to methlymalonic acid quantitation method. In our study, mass spectrometric detection was performed. Kara et al. [9] analyzed this parameter with flourometric detection. The number of the participants in our study was also higher compared to Kara et al. [9].

\section{Conclusion}

In conclusion, our results presented that serum sitosterol levels are significantly higher in patients with stroke compared to controls. Therefore, routine measurement of serum levels of sitosterol in patients with stroke might be considered to identify patients with possible sitosterolemia and for treatment. There were no significant differences for serum homocysteine, stigmasterol and Ox-LDL levels between the patient and control groups in our study.

\section{Conflict of interest}

The authors declared no conflict of interest.

\begin{abstract}
Abbreviations
ABC: ATP-binding cassette

Hcy: Homocysteine

Ox-LDL: oxidized LDL

UMMA: Urine methylmalonic acid

SMMA: Serum methylmalonic acid

LC-MS/MS: Liquid Chromatography Tandem Mass Spectrometry

GC-MS: Gas Chromatography-Mass Spectrometry
\end{abstract}

\section{Acknowledgements}

This research was supported by Selcuk University, Scientific Research Projects Department (BAP), (Project number, 13401130), Konya, Turkey.

\section{References}

1. De Jesús Llibre J, Valhuerdi A, Fernández O, Llibre JC, Porto R, López AM, et al. Prevalence of stroke and associated risk factors in older adults in Havana City and Matanzas Provinces, Cuba (10/66 population-based 
study). MEDICC Rev. 2010;12(3):20-6.

2. Harcombe Z, Baker JS. Plant sterols lower cholester$\mathrm{ol}$, but increase risk for coronary heart diseas. Online J Biol Sci. 2014;14(3):167-9. DOI: 10.3844/ojbsci.2014.167.169

3. Sudhop T, Bergmann KV. Sitosterolemia - a rare disease Are elevated plant sterols an additional risk factor? Z Kardiol. 2004;93:921-8. DOI: 10.1007/s00392-004$0165-2$

4. Igel M, Giesa U, Lutjohann D, von Bergmann K. Comparison of the intestinal uptake of cholesterol, plant sterols, and stanols in mice. J Lipid Res.2003;44(3):533-8. DOI: 10.1194/jlr.M200393-JLR200

5. Marangoni F, Poli A. Phytosterols and cardiovascular health. Pharmacol Res. 2010;61(3):193-9. DOI: 10.1016/j.phrs.2010.01.001

6. Párraga I, Torres JL, Andrés F, Navarro B, Campo JM, Reyes MG et al. Effect of plant sterols on the lipid profile ofpatients with hypercholesterolaemia. Randomised, experimental study. BMC Complement Altern Med. 2011;11:73:1-8.

7. Calpe-Berdiel L, Méndez-González J, Blanco-Vaca F, Carles Escolà-Gil J. Increased plasma levels of plant sterols and atherosclerosis: a controversial issue. Curr Atheroscler Rep. 2009;11(5):391-8. DOI: 10.1007/ s11883-009-0059-x

8. Yoo EG. Sitosterolemia: a review and update of pathophysiology, clinical spectrum, diagnosis, and management. Ann Pediatr Endocrinol Metab. 2016;21:7-14. DOI: 10.6065/apem.2016.21.1.7

9. Kara N, Senes M, Coskun O, Inan L, Saydam G, Yucel D. Urinary methylmalonic acid levels in patients with acute ischemic stroke. Clin Biochem. 2009;42(78):578-83. DOI: 10.1016/j.clinbiochem.2009.02.018

10. Kalita J, Kumar G, Bansal V, Misra UK. Relationship of homocysteine with other risk factors and outcome ofischemic stroke. Clin Neurol Neurosurg. 2009 May;111(4):364-7. DOI: 10.1016/j.clineuro. 2008.12 .010

11. Miettinen TA, Railo M, Lepantalo M, Gylling H. Plant sterols in serum and in atherosclerotic plaques of patients undergoing carotid endarterectomy. Aterosclerosis. 2005;45:1794-801. DOI: $10.1016 / j$. jacc.2005.02.063

12. Weinbrenner T, Cladellas M, Covas MI, Fito M, Tomas M, Senti M, et al. High oxidative stress in patients with stable coronary heart disease. Atherosclerosis. 2003;168:99-106. DOI: 10.1016/S00219150(03)00053-4

13. Ratnayake WMN, PlouffeL, Abbe MRL, Trick K, Mueller R, Hayward S. Comparative Health Effects of Margarines Fortified with PlantSterols and Stanols on a Rat Model for Hemorrhagic Stroke. Lipids. 2003;38:1237-47. DOI: 10.1007/s11745-003-1184-8
14. Nardi K, Leys D, Eusebi P, Cordonnier C, Gautier $\mathrm{S}$, Hénon H, Bordet R. Influence of lipid profiles on the risk of hemorrhagic transformation after ischemic stroke: systematic review. Cerebrovasc Dis Extra. 2011;1(1):130-41. DOI: 10.1159/000335014

15. Ahmida HS, Bertucci P, Franzò L, Massoud R, Cortese C, Lala A et al. Simultaneous determination of plasmatic phytosterols and cholesterol precursors using gas chromatography-mass spectrometry (GC-MS) with selective ion monitoring (SIM). J Chromatogr B Analyt Technol Biomed Life Sci. 2006;842:43-7. DOI: 10.1016/j.jchromb.2006.05.024

16. Rafii M, Elango R, Courtney-Martin G, House JD, Fisher L, Pencharz PB. High-throughput and simultaneous measurement of homocysteine and cysteine in human plasma and urine by liquid chromatography-electrospray tandem mass spectrometry. Anal Biochem. 2007;371(1):71-81. DOI: 10.1016/j.ab.2007.07.026

17. Kushnir MM, Komaromy-Hiller G, Shushan B, Urry FM, Roberts WL. Analysis of dicarboxylic acids by tandem mass spectrometry. High-throughput quantitative measurement of methylmalonic acid in serum, plasma, and urine. Clin Chem. 2001;47(11):1993-2002.

18. Alberici RM, Fernandes GD, Porcari AM, EberlinMN, Barrera-Arellano D, Fernández FM. Rapid fingerprinting of sterols and related compounds in vegetable and animal oils and phytosterol enriched- margarines by transmission mode direct analysis in real time mass spectrometry. Food Chem. 2016;211:661-8. DOI: 10.1016/j.foodchem.2016.05.057

19. Weingärtner O, Lütjohann D, Ji S, Weisshoff N, List F, Sudhop T et al. Vascular effects of diet supplementation with plant sterols. J Am Coll Cardiol. 2008;51(16):155361. DOI: $10.1016 /$ j.jacc.2007.09.074

20. Ogawa H, Yamamoto K, Kamisako T, Meguro T. Phytosterol additives increase blood pressure and promote stroke onset in salt-loaded stroke-prone spontaneously hypertensive rats. Clin Exp Pharmacol Physiol. 2003;30(12):919-24. DOI: 10.1111/j.14401681.2003.03939.x

21. Mannarino E, Pirro M, Cortese C, Lupattelli G, Siepi D, Mezzetti A et al. Effects of a phytosterol-enriched dairy product on lipids, sterols and 8-isoprostane in hypercholesterolemic patients: a multicenter Italian study. Nutr Metab Cardiovasc Dis. 2008;19(2):84-90. DOI: 10.1016/j.numecd.2008.03.012

22. Ganguly P, Alam SF.Role of homocysteine in the development of cardiovascular disease. Nutr J. 2015;14(6):110. DOI: $10.1186 / 1475-2891-14-6$

23. Xie R, Jia D, Gao C, Zhou J, Sui H, Wei X et al. Homocysteine induces procoagulant activity of red blood cells via phosphatidylserine exposure and microparticles generation. Amino Acids. 2014;46:1997-2004. DOI: $10.1007 / \mathrm{s} 00726-014-1755-6$ 